\title{
An Image Analysis of Breast Thermograms
}

\author{
Ryszard S. Choras \\ The Institute of Telecommunications and Computer Sciences, UTP University of Science and Technology, Bydgoszcz 85-796, Poland
}

\begin{abstract}
Thermography (infrared imaging) is a non-invasive technique applied for the detection breast cancer. We consider the problem of automatically recognition malignant breast from frontal view thermography image presented as gray scale image. This framework provides insights into several issues: breast Region of Interest (ROI) detection, extraction statistical features, extraction features based on texture and co-occurrence matrix.
\end{abstract}

Key words: Breast thermograms, feature extraction, Gabor wavelets, co-occurrence matrix, texture, Hu moments.

\section{Introduction}

Mammography is the technique that has the potential to detect very small and very early cancers in the breast. Thermography (also called infrared imaging) is a noninvasive, safe test that can detect breast cancer. Tumors require their own blood supply and thus make a lot of blood vessels in the area of a cancer. The increase in blood supply to feed a breast tumor causes the temperature of the surface of the breast to increase. Breast tumors radiate heat: they are faster-growing and have more vascularity than the surrounding normal tissue. Images are taken by a thermal sensitive camera to capture a digital image based on heat radiating from the body [9]. A computer-assisted interpretation of the digital image helps to determine whether a local abnormality in breast tissue temperature is present, which may indicate the presence of disease.

Each breast's image is placed into one of five thermobiological $(\mathrm{TH})$ categories (Figs. 1 and 2):

(a) $\mathrm{TH} 1$ - Normal uniform non-vascular;

(b) $T H 2$ - Normal uniform vascular;

(c) $\mathrm{TH} 3$-Equivocal (questionable);

(d) TH4-Abnormal;

(e) TH5-Severely abnormal.

Corresponding author: Ryszard S. Choras, Ph.D., DSc, professor, research fields: signal processing, image processing, computer vision. E-mail: choras@utp.edu.pl.
An infrared thermogram is an image of temperature distribution of the breast. Thermograms are viewed as anatomical images in temperature-related colors or grayscale. Each shade of color or gray represents a range of temperatures $[5,8]$.

In medicine, where the temperature range is more limited a "rainbow" palette is preferred, with red as hot and blue/black as cold (Fig. 3a). In case where a breast infrared thermogram is displayed in gray scale, the brighter pixels have higher temperature values (Fig. 3b). The thermal patterns of two normal, healthy breasts are equally symmetrical uniform temperature distributions [2].

\section{Preprocessing and Segmentation}

The image is represented as a function of two variables $(x, y)$. The image in its digital form is usually stored as a two-dimensional array. If $x=\{1,2, \ldots, M\}$ and $y=\{1,2, \ldots, N\}$ are the spatial domain, then $D=M \times N$ is the set of resolution cells and the digital image $F$ is a function which assigns some greytone value to each and every resolution cell, i.e., $F=M \times N \rightarrow G$.

$$
F=\{f(x, y) \mid(x, y) \in D \text { and } f(x, y) \in G\}
$$

For color image $f(x, y)$ denotes the color value at pixel $(x, y)$, i.e., $(x, y)=\left\{f_{R}(x, y), f_{G}(x, y), f_{B}(x, y)\right\}$.

For black and white images, $f(x, y)$ denotes the gray scale intensity value of pixel $(x, y)$. 


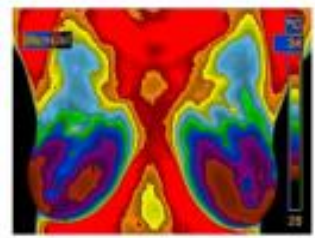

(a)

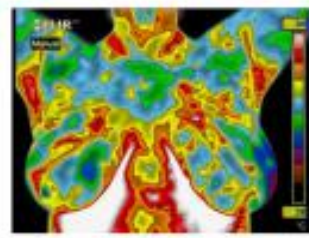

(b)

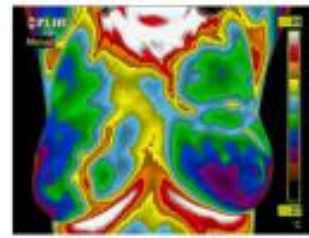

(c)

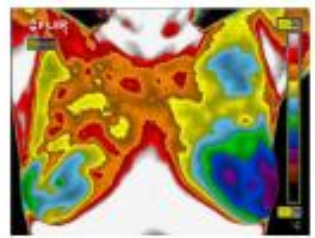

(d)

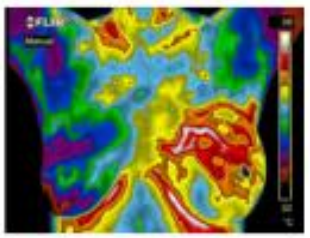

(e)

Fig. 1 Thermobiological $(\mathrm{TH})$ categories.

\begin{tabular}{|c|c|c|c|c|}
\hline $\begin{array}{c}\text { TH1 } \\
\text { lowest }\end{array}$ & $\begin{array}{l}\mathrm{TH} 2 \\
\text { low }\end{array}$ & $\begin{array}{c}\text { TH3 } \\
\text { medium }\end{array}$ & $\begin{array}{l}\text { TH4 } \\
\text { high }\end{array}$ & $\begin{array}{c}\text { TH5 } \\
\text { hlghest }\end{array}$ \\
\hline
\end{tabular}

Fig. 2 Risk level of the $T H$ categories.

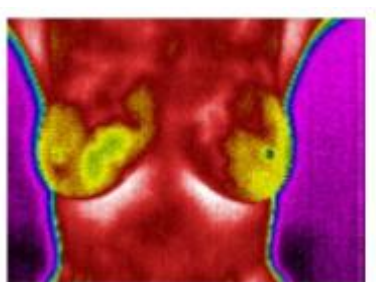

(a)

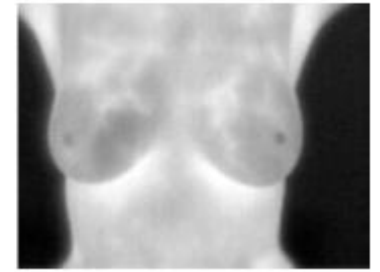

(b)
Fig. 3 Rainbow and grey level pallete.

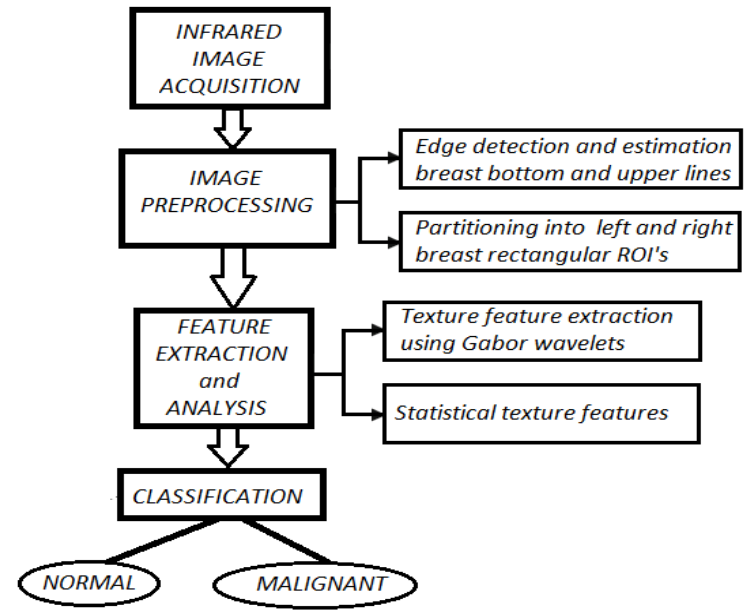

Fig. 4 The block diagram of the proposed method.

Fig. 4 shows the block diagram of the proposed method described in this paper.

An effective approach to automatically detect cancer cases is to study the symmetry between the left and right breast. When the images are relatively symmetrical, small asymmetries may indicate a suspicious region. The thermograms are first automatic segmented.

Segmentation steps are the followings:

(1) Contour detection using Canny algorithm;
(2) Estimation of the breast bottom line based on edge image and breast upper line based on curvature in left and right sides of body;

(3) Segment the left and right breasts rectangular ROI's.

Fig. 5 illustrates the described steps.

The size of left and right breast $R O I$ is $X \times Y$. The next stages intend to separate the left and the right breast image.

\section{Texture Feature from Gabor Wavelet Transform}

Gabor wavelet is a powerful tool to extract texture features and in the spatial domain is a complex exponential modulated by a Gaussian function [3]. In the most general the Gabor wavelet is defined as follows $[1,4]$ :

$$
\left.\operatorname{Gab}(x, y, \omega, \theta)=\frac{1}{2 \pi \sigma_{x} \sigma_{y}} e^{\left[-\frac{1}{2}\left(\left(\frac{x_{\theta}}{\sigma_{x}}\right)^{2}+\left(\frac{y_{\theta}}{\sigma_{y}}\right)^{2}\right)+j \omega x_{\theta}\right.}\right]_{(2)}
$$

where $j=\sqrt{-1}, \sigma_{x}$ and $\sigma_{y}$ are the scaling parameters of the filter, $\omega$ is the radial frequency of the sinusoid, $x_{\Theta}=x \cos \Theta+y \sin \Theta, y_{\Theta}=-x \sin \Theta+y \cos \Theta$ and $\Theta \in[0, \pi]$ specify the orientation of the Gabor filters.

By selecting different center frequencies and orientations, we can obtain a family of Gabor kernels, which can then be used to extract features from an image. Fig. 7 presents the real and imaginary parts of Gabor filters.

Gabor filtered output of the image is obtained by the convolution of the image with Gabor function for each of the orientation/spatial frequency (scale) orientation.

For pixel $F(x, y)$ in an image, its Gabor feature is treated as a convolution: 

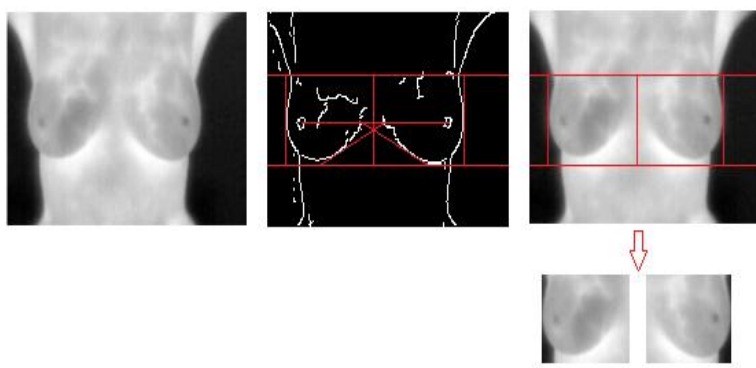

Fig. 5 Segmentation and $\mathrm{ROI}$ extraction. From left to right: Original grey image, Canny edge detection with $t h 1=43$ and $t h 2=78$ and left and the right $R O I$ breast image.

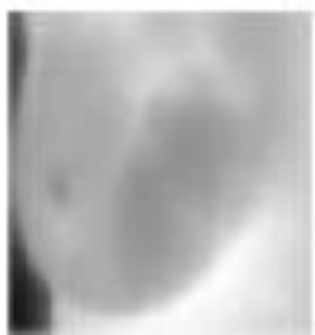

(a)

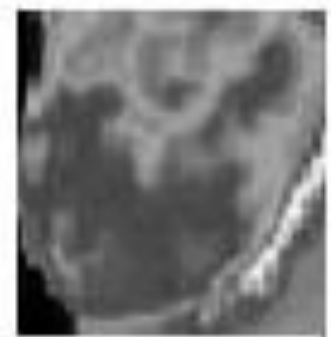

(c)

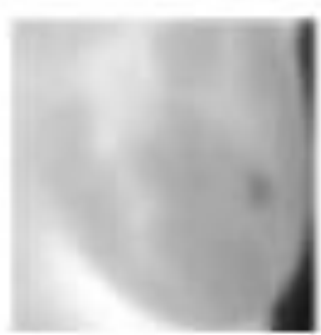

(b)

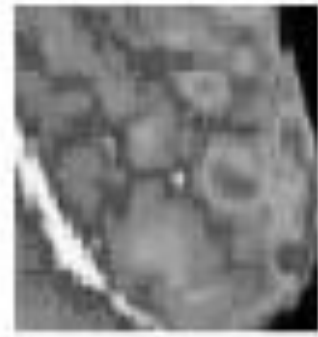

(d)

Fig. 6 Right and left $\mathrm{ROI}$ breast images-top normal images, below malignant images.
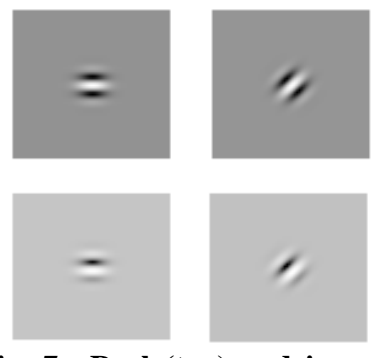

Fig. 7 Real (top)
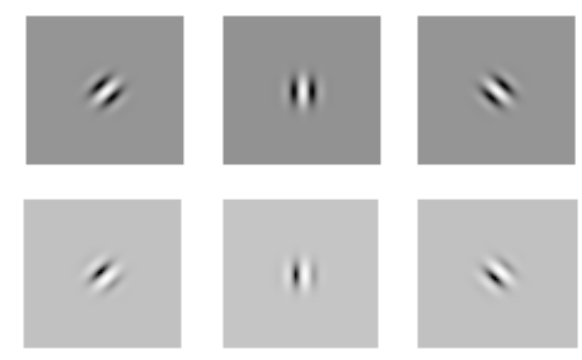

filters $\left(\theta=0^{\circ}, 45^{\circ}, 90^{\circ}, 135^{\circ}\right.$, scale $\left.=4\right)$.

$$
G_{\omega, \theta}(x, y)=F(x, y) * G a b(\omega, \theta, x, y)
$$

For $k$ frequencies and $l$ orientations, we have $k \cdot l$ complex coefficients for each image point.

Features based on the Gabor filters responses can be represented by [6]:

$$
\begin{gathered}
\mu(x, y)=\frac{1}{X Y} \sum_{x=1}^{X} \sum_{y=1}^{Y} G_{\omega, \theta}(x, y) \\
\operatorname{std}(x, y)=\sqrt{\sum_{x=1}^{X} \sum_{y=1}^{Y}\left(\left|G_{\omega, \theta}(x, y)\right|-\mu(x, y)\right)^{2}}(5) \\
\text { Skew }=\frac{1}{X Y} \sum_{x=1}^{X} \sum_{y=1}^{Y}\left(\frac{G_{\omega, \theta}(x, y)-\mu(x, y)}{s t d(x, y)}\right)^{3} \\
\text { Kurtosis }=\frac{1}{X Y} \sum_{x=1}^{X} \sum_{y=1}^{Y}\left(\frac{G_{\omega, \theta}(x, y)-\mu(x, y)}{s t d(x, y)}\right)^{4}
\end{gathered}
$$

where $X, Y$ is image dimension.

Table 1 presented these parameters for normal and malignant breast left and right ROI's images.

Additional features to describe asymmetry between left and right breast region are based on the co-occurrence matrixes $P_{d, \varphi}(x, y)$ [6].

The co-occurrence matrix $P_{d, \varphi}(x, y)$ counts the co-occurrence of pixels with gray values $i$ and $j$ at a given distance $d$. The distance $d$ is defined in polar coordinates $(d, \varphi)$, with discrete length and orientation. The co-occurrence matrix $P_{d, \varphi}(x, y)$ can now be defined as follows:
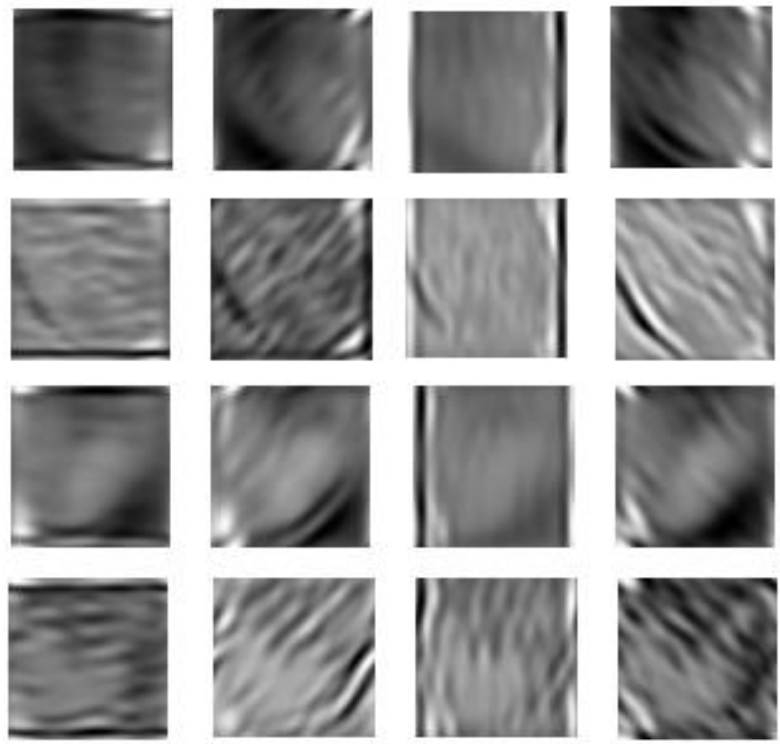

Fig. 8 Normal and malignant filtered breast $R O I$ 's images. First and third row - left and right breast normal ROI's image; second and fourth row - left and right breast malignant ROI's image.

$$
P_{d, \varphi}(x, y)=\operatorname{card}\left\{\begin{array}{c}
\left(\left(x_{1}, y_{1}\right),\left(x_{2}, y_{2}\right)\right) \in(X Y) \times(X Y) \\
f \text { or } f\left(x_{1}, y_{1}\right)=i, f\left(x_{2}, y_{2}\right)=j \\
\left(x_{2}, y_{2}\right)=\left(x_{1}, y_{1}\right)+(d \cos \varphi, d \sin \varphi) \\
\text { for } 0<i, j<G-1
\end{array}\right.
$$


where card $\{$.$\} denotes the number of elements in$ the set.

Let $G$ be the number of gray-values in the image, then the dimension of the co-occurrence matrix $P_{d, \varphi}(x, y)$ will be $G \times G$. So, the computational complexity of the co-occurrence matrix depends quadratically on the number of gray-scales used for quantization.

Features can be extracted from the co-occurrence matrix to reduce feature space dimensionality and the formal definitions of five features from the co-occurrence matrix are done.

The features are:

(1) Second Angular Moment

$$
S A M=\sum_{i=1}^{G} \sum_{j=1}^{G}\left[P_{d, \varphi}(x, y)\right]
$$

(2) Contrast

$$
\text { Con }=\sum_{i=1}^{G} \sum_{j=1}^{G}(x-y)^{2} P_{d, \varphi}(x, y)
$$

(3) Correlation

$$
\operatorname{Corr}=\frac{\sum_{i=1}^{G} \sum_{j=1}^{G}\left(\left[x y P_{d, \varphi}(x, y)\right]-\mu_{x} \mu_{y}\right)^{2}}{\sigma_{x} \sigma_{y}}
$$

(4) Inverse Differential moment

$$
I D M=\sum_{i=1}^{G} \sum_{j=1}^{G} \frac{P_{d, \varphi}(x, y)}{1+(x-y)^{2}}
$$

(5) Entropy

$$
E=-\sum_{i=1}^{G} \sum_{j=1}^{G} P_{d, \varphi}(x, y) \log P_{d, \varphi}(x, y)
$$

Table 2 shows these features for the left $(L B)$ and right $(R B)$ normal and malignant breast ROI's image.

\begin{tabular}{|c|c|c|c|c|c|c|}
\hline \multicolumn{7}{|c|}{ Benign breast image } \\
\hline \multirow{2}{*}{ Parameter } & \multicolumn{3}{|c|}{$L B R O I$ breast image } & \multicolumn{3}{|c|}{$R B R O I$ breast image } \\
\hline & std & Skew & Kurtosis & std & Skew & Kurtosis \\
\hline $0^{\circ}$ & 31.942 & 1.116 & 3.001 & 34.888 & 0.112 & 1.618 \\
\hline $45^{\circ}$ & 38.399 & 0.956 & 2.009 & 40.888 & -0.089 & 0.519 \\
\hline $90^{\circ}$ & 33.271 & 0.995 & 2.849 & 32,229 & 0.570 & 2.748 \\
\hline \multicolumn{7}{|c|}{ Malignant breast image } \\
\hline \multirow{2}{*}{ Parameter } & \multicolumn{3}{|c|}{$L B R O I$ breast image } & \multicolumn{3}{|c|}{$R B R O I$ breast image } \\
\hline & std & Skew & Kurtosis & std & Skew & Kurtosis \\
\hline $0^{\circ}$ & 27.196 & -0.563 & 4.467 & 31.302 & 0.159 & 1.687 \\
\hline $45^{\circ}$ & 29.761 & 0.614 & 2.792 & 34.048 & -0.543 & 1.044 \\
\hline $135^{\circ}$ & 31.987 & -0.540 & 2.871 & 38.194 & 0.343 & 0.220 \\
\hline
\end{tabular}

\section{Moment-Based Features}

The breast ROI's can be represented by the spatial

\begin{tabular}{|c|c|c|c|c|c|c|c|c|c|c|}
\hline \multicolumn{11}{|c|}{ Benign breast image } \\
\hline \multirow{2}{*}{ Parameter } & \multicolumn{5}{|c|}{$L B R O I$ breast image } & \multicolumn{5}{|c|}{$R B R O I$ breast image } \\
\hline & $A S M$ & Con & Corr & $I D M$ & $E$ & $A S M$ & Con & Corr & $I D M$ & $E$ \\
\hline$\overline{0^{\circ}}$ & 0.001 & 173.130 & $8.662 \mathrm{E}-4$ & 0.336 & 7.077 & 0.001 & 189.887 & $7.178 \mathrm{E}-4$ & 0.292 & 7.242 \\
\hline $45^{\circ}$ & $7.156 \mathrm{E}-4$ & 267.995 & $6.038 \mathrm{E}-4$ & 0.226 & 7.572 & $5.808 \mathrm{E}-4$ & 359.270 & $5.180 \mathrm{E}-4$ & 0.203 & 7.781 \\
\hline $90^{\circ}$ & 0.001 & 747.325 & $5.912 \mathrm{E}-4$ & 0.291 & 7.135 & 0.001 & 1107.871 & 4.513E-4 & 0.300 & 7.212 \\
\hline $135^{\circ}$ & $5.366 \mathrm{E}-4$ & 293.376 & 4.958E-4 & 0.202 & 7.851 & $5.755 \mathrm{E}-4$ & 301.321 & $5.407 \mathrm{E}-4$ & 0.199 & 7.769 \\
\hline \multicolumn{11}{|c|}{ Malignant breast image } \\
\hline \multirow{2}{*}{ Parameter } & \multicolumn{5}{|c|}{$L B$ ROI breast image } & \multicolumn{5}{|c|}{$R B$ ROI breast image } \\
\hline & $A S M$ & Con & Corr & $I D M$ & $E$ & $A S M$ & Con & Corr & $I D M$ & $E$ \\
\hline $0^{\circ}$ & 0.00 & 200.520 & 0.001 & 0.296 & 7.039 & 0.001 & 223.494 & $8.506 \mathrm{E}-4$ & 0.281 & 7.297 \\
\hline $45^{\circ}$ & $4.821 \mathrm{E}-4$ & 181.736 & $9.535 \mathrm{E}-4$ & 0.127 & 7.913 & 4.067E-4 & 616.283 & $5.860 \mathrm{E}-4$ & 0.116 & 8.099 \\
\hline $90^{\circ}$ & 0.001 & 654.125 & $6.230 \mathrm{E}-4$ & 0.191 & 7.360 & $4.257 \mathrm{E}-4$ & 613.156 & $6.718 \mathrm{E}-4$ & 0.112 & 8.043 \\
\hline $135^{\circ}$ & $4.676 \mathrm{E}-4$ & 470.031 & $6.726 \mathrm{E}-4$ & 0.122 & 7.998 & $3.513 \mathrm{E}-4$ & 357.147 & $5.827 \mathrm{E}-4$ & 0.110 & 8.191 \\
\hline
\end{tabular}
moments of its intensity function. In the spatial case,

Table 1 Texture parameters of the left (LB) and right (RB) breast ROI's image.

Table 2 Texture parameters of the left $(L B)$ and right $(R B)$ normal and malignant breast $R O I$ 's image. 
Table 3 Moment invariants for ROI's breast images with Fig. 6.

\begin{tabular}{|c|c|c|c|c|c|c|c|c|}
\hline \multirow{2}{*}{$\overbrace{\text { Hu moments }}^{\text {Image }} \varphi_{0}$} & \multicolumn{4}{|c|}{ Benign LB ROI's image } & \multicolumn{4}{|c|}{ Benign $R B$ ROI's image } \\
\hline & & $45^{\circ}$ & $90^{\circ}$ & $135^{\circ}$ & $0^{\circ}$ & $45^{\circ}$ & $90^{\circ}$ & $135^{\circ}$ \\
\hline$H u 1$ & 22.5758 & 21.6936 & 15.0337 & 20.3792 & 16.0810 & 14.2507 & 14.1583 & 16.6080 \\
\hline$H u 2$ & 0.6661 & 2.0352 & 0.3929 & 1.4409 & 1.9940 & 3.1830 & 0.6998 & 6.0098 \\
\hline Hu3 & 659.3907 & 867.8238 & 21.7467 & 866.7806 & 116.8156 & 91.5015 & 5.6585 & 173.0833 \\
\hline$H u 4$ & 460.0966 & 645.4099 & 61.4241 & 632.2971 & 45.8474 & 16.4694 & 9.2216 & 31.3254 \\
\hline Hu5 & -202361.7656 & -384947.75 & -2375.6889 & -352336.3750 & -2583.6867 & 759.1135 & -52.3741 & 2870.1812 \\
\hline Hu6 & -323.2109 & -855.6223 & -38.2720 & -696.7250 & -63.2667 & 8.3043 & -7.5937 & 30.7470 \\
\hline Hu7 & 369172.6875 & 747424.8126 & 4197.7706 & 9078.3751 & 412.1985 & -663.8193 & -13.1033 & -2026.007 \\
\hline \multicolumn{2}{|l|}{ Image } & \multicolumn{2}{|c|}{ Benign $L B$ ROI's image } & & \multicolumn{2}{|c|}{ Benign $R B$ ROI's image } & & \\
\hline Hu moments $\varphi$ & & $45^{\circ}$ & $90^{\circ}$ & $135^{\circ}$ & $0^{\circ}$ & $45^{\circ}$ & $90^{\circ}$ & $135^{\circ}$ \\
\hline$H u 1$ & 12.9861 & 16.9149 & 11.1434 & 10.7751 & 16.3185 & 11.7599 & 12.7305 & 17.6572 \\
\hline$H u 2$ & 0.0249 & 0.2066 & 0.0086 & 0.0086 & 0.1028 & 0.0582 & 0.1727 & 0.5507 \\
\hline$H u 3$ & 1.3818 & 15.3584 & 0.2102 & 0.9991 & 8.6339 & 1.3814 & 0.8360 & 35.0412 \\
\hline$H u 4$ & 17.7733 & 43.1753 & 2.7171 & 3.5471 & 66.2766 & 59.2879 & 82.8484 & 566.5087 \\
\hline Hu 5 & -33.9289 & -41.8188 & 1.6895 & 5.6020 & -1432.7243 & -478.3829 & -642.5955 & -63704.8906 \\
\hline Hu6 & 2.7766 & 13.7490 & 0.2326 & 0.3213 & -19.5379 & -8.0951 & -15.1976 & -139.0782 \\
\hline Hu7 & 117.6713 & 1647.1287 & -2.2362 & -7.4704 & -989.8900 & $-764 . .0012$ & -2182.9470 & -49387.6484 \\
\hline \multirow{2}{*}{\multicolumn{4}{|c|}{$\begin{array}{l}\qquad m_{p q}=\sum_{x=1}^{X} \sum_{y=1}^{Y} x^{p} y^{q} f(x, y) \\
\text { where } f(x, y) \text { is the intensity function representing the } \\
\text { image, the integration is over the entire image. } \\
\text { The central moments are given by } \\
\qquad m_{p q}=\sum_{x=1}^{X} \sum_{y=1}^{Y}\left(x-x_{0}\right)^{p}\left(y-y_{0}\right)^{q} f(x, y)(15)\end{array}$}} & \multicolumn{5}{|c|}{$H u 4=\left(\eta_{30}+\eta_{12}\right)^{2}+\left(\eta_{21}+\eta_{03}\right)^{2}$} \\
\hline & & & & \multicolumn{5}{|c|}{$\begin{array}{l}\text { Hu5 }=\left(\eta_{30}-3 \eta_{12}\right)\left(\eta_{30}+\eta_{12}\right) \times \\
\times\left[\left(\eta_{30}+\eta_{12}\right)^{2}-3\left(\eta_{21}+\eta_{03}\right)^{2}\right]+ \\
\quad+\left(3 \eta_{21}-\eta_{03}\right)\left(\eta_{21}+\eta_{03}\right) \times\end{array}$} \\
\hline \multicolumn{4}{|c|}{$x_{0}=\frac{m_{10}}{m_{00}}$ and $y_{0}=\frac{m_{01}}{m_{00}}$} & \multicolumn{5}{|c|}{$\begin{aligned} & +4 \eta_{11}\left(\eta_{30}+\eta_{12}\right)\left(\eta_{21}+\eta_{03}\right) \\
H u 7= & \left(3 \eta_{21}-\eta_{03}\right)\left(\eta_{30}+\eta_{12}\right)\end{aligned}$} \\
\hline Normalized centı & $\begin{array}{l}\text { tral moment } \\
=\frac{m_{p q}}{\left(m_{00}\right)^{\alpha}}, \alpha\end{array}$ & $\begin{array}{l}\mu_{p q} \\
=\frac{p+q}{2}+1\end{array}$ & $(17)$ & & $\begin{array}{r}{\left[\left(\eta_{30}+\eta\right.\right.} \\
-\left(\eta_{30}\right. \\
{\left[3\left(\eta_{30}+\right.\right.}\end{array}$ & $\begin{array}{l}12)^{2}-3(r \\
\left.-3 \eta_{12}\right)(\eta \\
\left.\eta_{12}\right)^{2}-(r\end{array}$ & $\begin{array}{l}\left.\eta_{21}+\eta_{03}\right)^{2} \\
\left.\eta_{21}+\eta_{03}\right) \\
\left.\eta_{21}+\eta_{03}\right)^{2}\end{array}$ & \\
\hline
\end{tabular}

Using nonlinear combinations of the lower order moments, a set of moment invariants (usually called geometric moments), which has the desirable properties of being invariant under translation, scaling and rotation, is derived. Hu [7] employed seven moment invariants that are invariant under rotation as well as translation and scale change, to recognize characters independently of their position size and orientation.

$$
\begin{gathered}
H u 1=\eta_{20}+\eta_{02} \\
H u 2=\left(\eta_{20}-\eta_{02}\right)^{2}+4 \eta_{11}^{2} \\
H u 3=\left(\eta_{30}-3 \eta_{12}\right)^{2}+\left(3 \eta_{21}-\eta_{03}\right)^{2}
\end{gathered}
$$

The results in terms of the $\mathrm{Hu}$ moment invariants for ROI's breast images with Fig. 6 are summarized in Table 3.

\section{Conclusion}

We have presented an approach to analyze breast thermography images. It is clear that Gabor wavelets, statistical texture parameters and invariant texture moments parameters discriminatingly detect benign and malignant breast cancer based on thermography gray scale images. Experimental results show that this approach has high classification accuracy. 


\section{References}

[1] Choras, R. S. 2007. "Image Feature Extraction Techniques and Their Applications for CBIR and Biometrics Systems." International Journal of Biology and Biomedical Engineering 1 (1): 6-16.

[2] Choras, R. S. 2015. "Analysis of Breast Thermography." The 2nd World Symposium on Web Applications and Networking 2015 (WSWAN 2015).

[3] Etehadtavakol, M., Ng, E. Y. K., Chandran, V., and Rabbani, H. 2013. "Separable and Non-separable Discrete Wavelet Transform Based Texture Features and Image Classification of Breast Thermograms." Infrared Physics \& Technology 61 (2013): 274-86
[4] Gabor, D. 1946. "Theory of Communication." J. Inst. Elect. Eng. 93: 429-59.

[5] Hammoud, R. I. (Ed.). 2009. Augmented Vision Perception in Infrared. Springer-Verlag.

[6] Haralick, R. M. 1979. "Statistical and Structural Approaches to Texture." IEEE Transaction on Systems, Man and Cybernetics 67: 786-804.

[7] Hu, M. K. 1962. "Visual Pattern Recognition by Moment Invariants." IRE Trans. Info. Theory IT-8: 179-87.

[8] Ring, E. F. J., and Ammer, K. 2012. "Infrared Thermal Imaging in Medicine." Physiological Measurement 33 (2012): R33-R46.

[9] Rogalski, A. 2011. "Recent Progress in Infrared Detector Technologies." Infrared Physics \& Technology 54 (2011): 136-54. 